\title{
Can a light ray distinguish charge of a black hole in nonlinear electrodynamics?
}

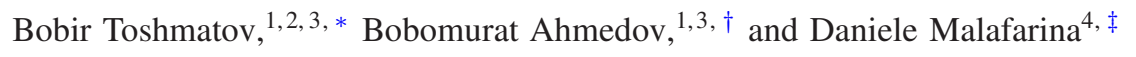 \\ ${ }^{1}$ Ulugh Beg Astronomical Institute, Astronomy 33, Tashkent 100052, Uzbekistan \\ ${ }^{2}$ Webster University in Tashkent, Alisher Navoiy 13, Tashkent 100011, Uzbekistan \\ ${ }^{3}$ Tashkent Institute of Irrigation and Agricultural Mechanization Engineers, Kori Niyoziy 39, Tashkent 100000, Uzbekistan \\ ${ }^{4}$ Department of Physics, Nazarbayev University, 53 Kabanbay Batyr, 010000 Nur-Sultan, Kazakhstan
}

\begin{abstract}
It is a well-known fact that light rays do not follow the null geodesics of the space-time in nonlinear electrodynamics; instead, they follow the null geodesics of the so-called effective space-time. Taking this into account, in this paper, we aim to discuss the possibility of distinguishing the type of charge with which the black hole is endowed, via the motion of light rays. The results show that, for any black hole being a charged solution of the field equations of general relativity coupled to the nonlinear electrodynamics, one cannot distinguish the two types of charge (magnetic or electric) through the motion of light rays around it.
\end{abstract}

\section{INTRODUCTION}

Despite the fact that Maxwell (linear) electrodynamics and general relativity are completely different classical theories, they both endure the so-called singularity problem, i.e. the fact that physically viable solutions of the theory generically exhibit singularities. The problem is more pronounced in general relativity, where the occurrence of curvature singularities disrupts the causal structure of the space-time. However, ideally, it would be preferable to develop classical theories for gravitation and electromagnetism that do not present singularities. It is clear that the solution of the singularity problem requires us to extend beyond classical general relativity and Maxwell electrodynamics, since these theories cannot avoid or solve the problem on their own.

In electrodynamics, experiments direct us to consider the Lagrangian density of the field to be approximately linear. In the simplest case, it is exactly linear so that if the field equations are solved, together with the Lorentz gauge, one will end up with the well-known Maxwell equations [1]. The validity of Maxwell's equations at the classical level have been widely demonstrated from experimental physics. However, theoretically, if these equations are solved for point charges, one would obtain diverging field quantities at the location of the point charge, a fact that is already quite inexplicable, which in turn results in infinite total energy for the electric field of a point charge, which clearly is physically undesirable.

General relativity also allows for the existence of spacetime singularities. Of course, not all singularities appearing in solutions of Einstein's equations are physical: For example, coordinate singularities that are defined by the divergence of one of the metric functions, may be merely mathematical, i.e. they can be eliminated with an appropriate coordinate transformation. However, curvature singularities, defined by the divergence of curvature invariants such as the Kretschmann scalar, cannot be eliminated by any change of coordinates and they are an intrinsic feature of the geometry. The singularity theorems show that such curvature singularities are an in-

\footnotetext{
toshmatov@astrin.ud

† ahmedov@astrin.uz

‡ daniele.malafarina@nu.edu.kz
}

evitable outcome of physically viable scenarios such as, for example, the dynamical collapse that leads to the formation of a black hole [2], thus making the problem of their resolution an important piece of the hunt for a better theory of gravity and of our understanding of extreme astrphysical phenomena [3]. The existence of curvature singularities is still one of the unsolved problems of the theory. Many approaches have been taken in the attempt to avoid this 'space-time pathology' and one of the most promising worked-out methods is based on coupling general relativity to nonlinear electrodynamics 四 7. These solutions can be of electrically, magnetically or dyonically (i.e. simultaneously electrically and magnetically) charged black holes [313, 31], like in the case of general relativity coupled to the linear electrodynamics that results the Reissner-Nordström solution.

Other new interesting phenomena appear in nonlinear electrodynamics. One of the such phenomena is associated with the propagation of light rays. It is a well-known fact that electromagnetic waves propagate along null geodesics of the space-time in vacuum and linear electrodynamics. However, this is not the case if the electromagnetic field is selfinteracting as in the case of nonlinear electrodynamics. Then light rays do not follow the null geodesics of a given spacetime metric, instead the paths of light can be described in terms of an effective space-time metric which represents a modification of the original space-time [14 25. This phenomenon can also be shown from perturbations theory, as in the high energy limit, the effective potential of the electromagnetic perturbations of the black hole in nonlinear electrodynamics coincides with the one governing the photon motion in the field of a central object [26-30].

Motivated by the peculiar phenomena of nonlinear electrodynamics discussed above, i.e. that light rays do not follow null geodesics, in this paper we aim to determine whether it is possible to distinguish the type of charge of the black hole from the motion of light rays in the given geometry. To do so, we consider a given spherically symmetric, static space-time that is either of electrically charged or magnetically charged black hole. By constructing the effective metrics for the geometry and studying the motion of light rays in these two space-times, we establish the criteria necessary to distinguish the two types of charges from the motion of photons. We show that it is not possible to distinguish the two types of 
charges only from the motion of light rays around the black hole. The paper is organized as follows: In Sec. II we briefly review the main equations to construct black hole solutions in general relativity coupled to nonlinear electrodynamics. In Sec. III we derive the effective metrics of the electrically and magnetically charged black holes and in Sec. IV we study the motion of light rays in these space-times. Finally, in Sec. V we discuss and summarize results. Throughout the paper, we adopt the following signature convention $(-,+,+,+)$ for the space-time metric and make use of natural units, thus setting $c=\hbar=G=1$.

\section{BASIC EQUATIONS}

A generic theory of general relativity coupled to nonlinear electrodynamics is characterized by the action

$$
S=\int d^{4} x \sqrt{-g} \mathcal{L},
$$

with the Lagrangian density given by

$$
\mathcal{L}=\frac{1}{16 \pi}[R-\mathscr{L}(F)],
$$

where $g$ and $R$ are the determinant of the metric tensor and the Ricci scalar, respectively while $\mathscr{L}$ is the Lagrangian density describing the nonlinear electrodynamics theory, which is a function of the Faraday tensor $F^{\mu \nu}$ through $F \equiv F_{\mu \nu} F^{\mu \nu}$. The electromagnetic field tensor satisfies $F_{\mu \nu}=\partial_{\mu} A_{\nu}-$ $\partial_{\nu} A_{\mu}$, with $A^{\mu}$ being the 4-potential. Since the Faraday tensor, $F_{\mu \nu}$, is antisymmetric, it has only six nonvanishing components: i.e. three for the electric field and three for the magnetic field.

To construct a solution of the theory described by (1), one needs to solve Einstein's field equations given by

$$
G_{\mu \nu}=T_{\mu \nu},
$$

where $G_{\mu \nu}$ is the Einstein tensor and $T_{\mu \nu}$ is the energymomentum tensor of the nonlinear electrodynamics field. Note that, for simplicity, in equation (3) the coefficient $8 \pi$ has been absorbed in the energy-momentum tensor. The energymomentum tensor of nonlinear electrodynamics is given by

$$
T_{\mu \nu}=2\left(\mathscr{L}_{F} F_{\mu}{ }^{\alpha} F_{\nu \alpha}-\frac{1}{4} g_{\mu \nu} \mathscr{L}\right),
$$

where $\mathscr{L}_{F}=\partial_{F} \mathscr{L}$. At the same time, the electromagnetic field is governed by Maxwell's equations for nonlinear electrodynamics, which can be written as

$$
\nabla_{\nu}\left(\mathscr{L}_{F} F^{\mu \nu}\right)=0, \quad \nabla_{\nu}{ }^{*} F^{\mu \nu}=0
$$

Where $* F^{\mu \nu}=\varepsilon^{\mu \nu \alpha \beta} F_{\alpha \beta} / 2$ is the dual electromagnetic strength tensor. The electromagnetic 4-potential can be written in spherical coordinates $\{t, r, \theta, \phi\}$ in the following form:

$$
A_{\mu}=\varphi(r) \delta_{\mu}^{t}-Q_{m} \cos \theta \delta_{\mu}^{\phi},
$$

where $\varphi(r)$ and $Q_{m}$ are the electric potential and total magnetic charge, respectively. The exterior of spherically symmetric, static, electrically and magnetically charged compact objects is described by the same line elements which can be written in general as

$$
d s^{2}=-f(r) d t^{2}+\frac{d r^{2}}{f(r)}+r^{2} d \Omega^{2},
$$

where $d \Omega^{2}=d \theta^{2}+\sin ^{2} \theta d \phi^{2}$ is the metric on the unit twosphere and the metric function $f(r)$ is given in the parameterized form as

$$
f(r)=1-\frac{2 m(r)}{r}
$$

with the mass function, $m(r)$ determined by the Lagrangian density of the nonlinear electrodynamics. In the absence of the electromagnetic field, the mass function takes constant value $m(r)=M$, consistent with the description of a purely gravitational mass. For the sake of our further calculations, here below we will briefly review the main points of the formalism for deriving electrically and magnetically charged black hole solutions.

\section{A. Electrically charged solution}

If the space-time is electrically charged, then the 4-potential of the electromagnetic field is given solely by the first term in equation (6), as $A_{t}=\varphi(r)$. The electromagnetic field 2-form can be written as

$$
\mathbf{F}_{2}=\varphi^{\prime}(r) \mathbf{d} r \wedge \mathbf{d} t
$$

Note that $F=-2 \varphi^{\prime 2}$. By solving the non linear Maxwell's equations (5), we arrive at the expression for the total electric charge inside a sphere with radius $r$

$$
Q_{e}=r^{2} \mathscr{L}_{F} \varphi^{\prime}
$$

At this point, to construct a solution, we need to solve Einstein's equations (3), which, for this system, reduce to only two independent equations. By solving them, we obtain the following:

$$
\begin{aligned}
& \mathscr{L}=\frac{2 m^{\prime \prime}}{r}, \\
& \mathscr{L}_{F}=\frac{2 m^{\prime}-r m^{\prime \prime}}{2 r^{2} \varphi^{\prime 2}} .
\end{aligned}
$$

By using (10) and 12 , we find the expression for the scalar electric potential as

$$
\varphi=\frac{3 m-r m^{\prime}}{2 Q_{e}} .
$$

If the mass function is constant, then, we immediately recover the Schwarzschild solution. If the electromagnetic field is linear, i.e., the Lagrangian density is linear function of $F$, by solving eqs. (11), (12), and (13), one can find the ReissnerNordström solution with mass function $m(r)=M-Q_{e}^{2} / 2 r$, and the Coulomb potential $\varphi \sim Q_{e} / r$. 


\section{B. Magnetically charged solution}

If the black hole is magnetically charged, then the 4potential of the electromagnetic field is given by the second term in equation (6), as $A_{\phi}=-Q_{m} \cos \theta$. The electromagnetic field 2-form can then be written as

$$
\mathbf{F}_{2}=Q_{m} \sin \theta \mathbf{d} \theta \wedge \mathbf{d} \phi .
$$

Note the electromagnetic field strength is $F=2 Q_{m}^{2} / r^{4}$. By solving Einstein's equations (3), we obtain the following expressions for the Lagrangian density:

$$
\begin{aligned}
& \mathscr{L}=\frac{4 m^{\prime}}{r^{2}}, \\
& \mathscr{L}_{F}=\frac{r^{2}\left(2 m^{\prime}-r m^{\prime \prime}\right)}{2 Q_{m}^{2}} .
\end{aligned}
$$

In the case that the electromagnetic field is linear, i.e. for Maxwell's theory, we have that $\mathscr{L}=F$ and by solving the above equations, we arrive at the mass function $m=$ $M-Q_{m}^{2} / 2 r$ that again represents the Reissner-Nordström space-time with a magnetic charge. We see that in the case of linear electrodynamics the two charges are not distinguishable in Reissner-Nordström's solution. On the other hand, in the nonlinear theory the two cases produce two different effective geometries. We shall now investigate whether such space-times may be distinguished by looking at the trajectories of light rays.

\section{EFFECTIVE METRICS}

As we mentioned in the case where the line element (7) is a solution of the field equations for general relativity coupled to nonlinear electrodynamics, light rays do not propagate along the null geodesics of the space-time metric, instead, they follow the null geodesics of the effective metric obtained from the metric tensor [8, 14]

$$
g_{\text {eff }}^{\mu \nu}=\mathscr{L}_{F} g^{\mu \nu}-\mathscr{L}_{F F} F_{\lambda}{ }^{\mu} F^{\lambda \nu} .
$$

\section{A. Electrically charged case}

In the electrically charged case the effective metric is written in the following form:

$$
d s^{2}=-\frac{f(r)}{\Phi} d t^{2}+\frac{1}{\Phi f(r)} d r^{2}+\frac{r^{2}}{\mathscr{L}_{F}} d \Omega^{2},
$$

where $\Phi=\mathscr{L}_{F}+2 F \mathscr{L}_{F F}$. When written in terms of the mass function $m(r)$, then the above line element takes the following form:

$$
\begin{aligned}
d s^{2}= & -\frac{r^{2}(r-2 m)\left(r m^{\prime \prime \prime}-m^{\prime \prime}\right)}{4 Q_{e}^{2}} d t^{2}+ \\
& +\frac{r^{4}\left(r m^{\prime \prime \prime}-m^{\prime \prime}\right)}{4 Q_{e}^{2}(r-2 m)} d r^{2}+\frac{r^{4}\left(2 m^{\prime}-r m^{\prime \prime}\right)}{2 Q_{e}^{2}} d \Omega^{2} .
\end{aligned}
$$

\section{B. Magnetically charged case}

On the other hand, the effective metric for a magnetically charged black hole is written in the following form:

$$
d s^{2}=-\frac{f(r)}{\mathscr{L}_{F}} d t^{2}+\frac{1}{\mathscr{L}_{F} f(r)} d r^{2}+\frac{r^{2}}{\Phi} d \Omega^{2},
$$

where again $\Phi=\mathscr{L}_{F}+2 F \mathscr{L}_{F F}$. And again, when written in terms of the mass function $m(r)$, it takes the following form:

$$
\begin{aligned}
d s^{2}= & -\frac{2 Q_{m}^{2}(r-2 m)}{r^{3}\left(2 m^{\prime}-r m^{\prime \prime}\right)} d t^{2}+ \\
& +\frac{2 Q_{m}^{2}}{r(r-2 m)\left(2 m^{\prime}-r m^{\prime \prime}\right)} d r^{2}+\frac{4 Q_{m}^{2}}{r^{2} m^{\prime \prime \prime}-r m^{\prime \prime}} d \Omega^{2} .
\end{aligned}
$$

We see that for a generic mass function $m(r)$ the effective metric of an electrically charged black hole (19) differs from that of a magnetically charged one (21).

\section{LIGHT RINGS AND GRAVITATIONAL LENSING}

Before finding the equations governing the motion of light rays in the effective space-time metrics for the electrically and magnetically charged cases, one may write the effective metrics (19) and (21) in the following unified form:

$$
d s^{2}=-A(r) d t^{2}+B(r) d r^{2}+C(r) d \Omega^{2} .
$$

Taking into account the symmetry of the space-time, one can easily notice that the momenta $p^{\mu}$ corresponding to time, $t$, and azimuthal angle, $\phi$, are conserved. These are related to the energy, E, and angular momentum, L, of test particles and photons. Restricting the attention to motion in the equatorial plane, $\theta=\pi / 2$, the conserved quantities are given by

$$
\mathrm{E}=A \dot{t}, \quad \mathrm{~L}=C \dot{\phi} .
$$

Since for photon's motion we have $p^{\mu} p_{\mu}=0$, the radial component of the 4-velocity of light rays can be written in terms of the conserved quantities as

$$
\dot{r}^{2}=\frac{1}{A B}\left(\mathrm{E}^{2}-\mathrm{V}_{\mathrm{eff}}\right), \quad \text { with } \quad \mathrm{V}_{\mathrm{eff}}=\mathrm{L}^{2} \frac{A}{C} .
$$

Then circular null geodesics are obtained by imposing $\dot{r}=$ $0=\ddot{r}$. Therefore, setting to zero the expression inside parenthesis in (24), one can find that the energy for photons on circular orbits, while the radius of circular null geodesics i.e., the light ring, is determined by the radius for which $\ddot{r}=0$, corresponding to the solution of the following equation:

$$
A C^{\prime}-A^{\prime} C=0 \text {. }
$$

Before turning to the further relativistic effects, let us consider the effective potentials in terms of the effective metrics of the electrically and magnetically charged black holes which are given by equations $(19)$ and $(21)$. 
In the case of the Reissner-Nordström black hole with mass function $m=M-Q^{2} / 2 r$, we recover the effective potential $V_{\text {eff }}=\left(1-2 M / r+Q^{2} / r^{2}\right) \mathrm{L}^{2} / r^{2}$. As we have mentioned in section II, for linear electrodynamics, the ReissnerNordström solution describes both electrically as well as magnetically charged black hole space-times via the same line element $(7)$. In other words, the mass functions for electrically and magnetically charged black holes in linear electrodynamics coincide. From equations (19) and (21) it is easy to realize that even though the effective metrics differ, the effective potentials for the motion of light rays in the electrically and magnetically charged cases are the same. In fact, the effective potentials from equation (24) in terms of the effective metrics of the electrically (19) and magnetically (21) charged black holes take the form

$$
\mathrm{V}_{\mathrm{eff}}=\frac{f\left(m^{\prime \prime}-r m^{\prime \prime \prime}\right)}{2 r\left(r m^{\prime \prime}-2 m^{\prime}\right)} \mathrm{L}^{2}
$$

Consequently, the radii of the light rings (25) are identical and it is not possible to distinguish the electrically charged from the magnetically charged case solely from the location of the photon sphere.

Despite the above result that the expression inside the parenthesis in equation 24 is identical for both electrically and magnetically charged cases, the product of the metric functions $A$ and $B$ differs in the two cases which suggests that there may be other ways of distinguishing the two charges from the motion of photons. In fact in the electrically charged case we get

$$
A B=\frac{r^{6}\left(m^{\prime \prime}-r m^{\prime \prime \prime}\right)^{2}}{16 Q_{e}^{4}}
$$

while for the magnetically charged case we have

$$
A B=\frac{4 Q_{m}^{4}}{r^{4}\left(r m^{\prime \prime}-2 m^{\prime}\right)^{2}} .
$$

In principle, considering other relativistic effects where these terms play a role may allow to distinguish the type of charge of the black hole. With this objective in mind, below we will consider gravitational lensing in the strong field regime of both space-times. As it was mentioned before, even though electrically and magnetically charged black holes in nonlinear electrodynamics are described by the same line element, their effective metrics differ and light rays follow the null geodesics of the effective space-time. Let us then consider light rays passing close to a compact, massive object and evaluate how light rays deviate from a straight trajectory while following the geodesics of the space-time surrounding a massive compact object.

We first derive the equation for the deflection angle of gravitational lensing in a generic spherically symmetric space-time given by equation (22). By using the equations of motion (23) and (24), we find the deflection angle of the light ray from

$$
\frac{d \phi}{d r}=\frac{\sqrt{B}}{\sqrt{C} \sqrt{\frac{A_{0}}{C_{0}} \frac{C}{A}-1}},
$$

where we have denoted the light ring solution of equation (25) with $r_{0}$ and consequently quantities $X$ evaluated at $r_{0}$ are indicated via the subscript $X_{0}$. One can easily notice that as the radius $r$ tends to $r_{0}\left(r \rightarrow r_{0}\right)$, the deflection angle diverges ? This fact shows that at $r_{0}$ light rays move along circular orbits.

Another interesting relativistic effect associated with gravitational lensing is the apparent time delay in the propagation of light rays passing near a massive object, i.e. the delay in travel time of the light ray from the source to the receiver. The time delay is found by using the equations of motion (23) and (24) and it can be written as

$$
\frac{d t}{d r}=\frac{\sqrt{B}}{\sqrt{A} \sqrt{1-\frac{C_{0}}{A_{0}} \frac{A}{C}}}
$$

From equation (30) we can see that when the turning point of the light ray reaches the light ring of the black hole at radius $r_{0}$, the delaying time diverges and the light ray never reaches the observer.

Now let us evaluate the deflection of light rays and the time delay due to the gravitational lensing for a central object that is either electrically or magnetically charged.

\section{A. Electrically charged case}

Rewriting the expressions (29) and (30) in terms of the effective metrics of the electrically charged black hole (19) we obtain

$$
\begin{aligned}
& \frac{d \phi}{d r}=\sqrt{\frac{z}{2 f}} \frac{1}{\sqrt{\frac{f_{0} z_{0}}{f z}-1}}, \\
& \frac{d t}{d r}=\frac{1}{f \sqrt{1-\frac{f z}{f_{0} z_{0}}}},
\end{aligned}
$$

where

$$
z=\frac{m^{\prime \prime}-r m^{\prime \prime \prime}}{r\left(r m^{\prime \prime}-2 m^{\prime}\right)}
$$

and the zero subscript indicates the value of a function evaluated at the light ring $r_{0}$.

\section{B. Magnetically charged case}

On the other hand, in terms of the effective metrics of the magnetically charged black hole (21), expressions (29) and

${ }^{1}$ The deflection angle of light rays is determined by solving the following
integral: $\Delta \phi\left(r_{0}\right)=2 \int_{r_{0}}^{\infty} d r\left(\frac{d \phi}{d r}\right)-\pi$. 
(30) take the following form:

$$
\begin{aligned}
& \frac{d \phi}{d r}=\sqrt{\frac{z}{2 f}} \frac{1}{\sqrt{\frac{f_{0} z_{0}}{f z}-1}}, \\
& \frac{d t}{d r}=\frac{1}{f \sqrt{1-\frac{f z}{f_{0} z_{0}}}},
\end{aligned}
$$

Interestingly, despite the fact that the equations of motion governing the propagation of light rays in the electrically and magnetically charged black hole space-times are different, the trajectories followed by light rays near a massive compact object coincide. Therefore, we conclude that it is not possible to distinguish an electrically charged black hole from a magnetically charged one just by measuring the deflection of light rays.

\section{DISCUSSION}

We have shown that, despite the fact that the effective metrics of electrically and magnetically charged black holes in general relativity coupled to nonlinear electrodynamics are different, photons follow the same trajectories and therefore, the observation of light propagation alone can not distinguish the two kinds of charges. Therefore, to gain some further insights it may be worth to return to the field equations presented in section II and analyze their dependence on the non linear electromagnetic Lagrangian term. In the electrically charged case, if $\mathscr{L}_{F}$ in (12) is written in terms of the total electrical charge of the black hole (10), and compared to the one (16) for the magnetically charged case, one would easily notice the following relation:

$$
\left(\mathscr{L}_{F}\right)_{e}=\frac{1}{\left(\mathscr{L}_{F}\right)_{m}}
$$

Moreover, it is easy to show that the following relation between the electromagnetic field strengths in both cases holds:

$$
\left(F \mathscr{L}_{F}^{2}\right)_{e}=-F_{m}
$$

If the effective metrics in equations (18) and (20) for both cases are rewritten taking into account the relations given above and considering the fact that the conformal factor does not affect the motion of light rays [32], one can easily obtain the following relation:

$$
\left(\frac{\mathscr{L}_{F}}{\Phi}\right)_{e}=\left(\frac{\Phi}{\mathscr{L}_{F}}\right)_{m},
$$

and consequently, end up with the identical space-times.

Based on the fact that the light ray does not follow null geodesics in general relativity coupled to nonlinear electrodynamics [14 16], instead they follow the null geodesics of the effective metric, in this paper we have studied the possibility that the type of charge with which the black hole space-time in endowed may be distinguished from the motion of light rays in the equatorial plane of a black hole geometry. To this aim, we considered that a space-time metric (7) with metric function (8) that is a solution of the field equations of general relativity minimally coupled to the nonlinear electrodynamics and describes a static and spherically symmetric black hole. Then, we considered separately the two cases in which the black hole is endowed with an electrical or a magnetic charge and studied the physical effects related to the propagation of light rays. We have shown that light rays follow the same trajectory in both cases, despite of the fact that the corresponding effective metrics are different. Therefore the observation of photon trajectories alone is not able to distinguish the two kinds of charges.

\section{ACKNOWLEDGEMENTS}

BT and BA acknowledge the support of Ministry of Innovative Development of the Republic of Uzbekistan Grants No. VA-FA-F-2-008 and No. MRB-AN2019-29. DM is supported by the Ministry of Education of the Republic of Kazakhstan's target program IRN: BR05236454 and Nazarbayev University Faculty Development Competitive Research Grant No. 090118FD5348.
[1] L. D. Landau and E. M. Lifshitz, The classical theory of fields (1975).

[2] R. Penrose, Phys. Rev. Lett. 14, 57 (1965),

[3] D. Malafarina, Universe 3, 48 (2017), arXiv:1703.04138 [grqc].

[4] E. Ayón-Beato and A. García, Phys. Rev. Lett. 80, 5056 (1998) gr-qc/9911046.

[5] E. Ayón-Beato and A. García, Phys. Lett. B 493, 149 (2000) gr-qc/0009077.

[6] K. A. Bronnikov, Phys. Rev. Lett. 85, 4641 (2000)

[7] I. Dymnikova. Classical and Quantum Gravity 21, 4417 (2004) gr-qc/0407072

[8] K. A. Bronnikov, Phys. Rev. D 63. 044005 (2001), grqc/0006014.
[9] Z.-Y. Fan and X. Wang, Phys. Rev. D 94, 124027 (2016), arXiv:1610.02636 [gr-qc].

[10] K. A. Bronnikov, Phys. Rev. D 96, 128501 (2017) arXiv: 1712.04342 [gr-qc]

[11] K. A. Bronnikov, Gravitation and Cosmology 23, 343 (2017) arXiv: 1708.08125 [gr-qc].

[12] B. Toshmatov, Z. Stuchlík, and B. Ahmedov, Phys. Rev. D 98, 028501 (2018), arXiv:1807.09502 [gr-qc].

[13] S. I. Kruglov, Gravitation and Cosmology 25, 190 (2019) arXiv:1909.05674 [gr-qc].

[14] M. Novello, V. A. De Lorenci, J. M. Salim, and R. Klippert, Phys. Rev. D 61, 045001 (2000), gr-qc/9911085.

[15] M. Novello, J. M. Salim, V. A. De Lorenci, and E. Elbaz, Phys. Rev. D 63, 103516 (2001). 
[16] Y. N. Obukhov and G. F. Rubilar, Phys. Rev. D 66, 024042 (2002), gr-qc/0204028.

[17] N. Bretón, Phys. Rev. D 72, 044015 (2005), hep-th/0502217.

[18] J. Schee and Z. Stuchlík, Classical and Quantum Gravity 33, 085004 (2016), arXiv:1604.00632 [gr-qc].

[19] Z. Stuchlík and J. Schee, Int. J. Mod. Phys. D 24, 1550020-289 (2015), arXiv:1501.00015 [astro-ph.HE].

[20] J. Schee and Z. Stuchlík, J. Cosmol. Astropart. Phys. 2015, 048 (2015), arXiv:1501.00835 [astro-ph.HE].

[21] J. Schee and Z. Stuchlík, Eur. Phys. J. C 79, 988 (2019), arXiv:1908.07197 [gr-qc].

[22] Z. Stuchlík, J. Schee, and D. Ovchinnikov, Astrophys. J. 887, 145 (2019).

[23] J. Schee and Z. Stuchlík, Astrophys. J. 874. 12 (2019).

[24] Z. Stuchlík and J. Schee, Eur. Phys. J. C 79, 44 (2019).

[25] R. A. Konoplya, J. Schee, and D. Ovchinnikov, arXiv e-prints , arXiv:2008.04118 (2020), arXiv:2008.04118 [gr-qc].
[26] C. Moreno and O. Sarbach, Phys. Rev. D 67, 024028 (2003), gr-qc/0208090

[27] B. Toshmatov, Z. Stuchlík, J. Schee, and B. Ahmedov, Phys. Rev. D 97, 084058 (2018), arXiv:1805.00240 [gr-qc].

[28] J. Li, K. Lin, and N. Yang, Eur. Phys. J. C 75, 131 (2015), arXiv: 1409.5988 [gr-qc]

[29] B. Toshmatov, Z. Stuchlík, and B. Ahmedov, Phys. Rev. D 98, 085021 (2018), arXiv:1810.06383 [gr-qc]

[30] B. Toshmatov, Z. Stuchlík, B. Ahmedov, and D. Malafarina, Phys. Rev. D 99, 064043 (2019), arXiv:1903.03778 [gr-qc].

[31] B. Toshmatov, Z. Stuchlík, and B. Ahmedov, Phys. Rev. D 95, 084037 (2017), arXiv:1704.07300 [gr-qc].

[32] B. Toshmatov, C. Bambi, B. Ahmedov, Z. Stuchlík, and J. Schee, Phys. Rev. D 96, 064028 (2017), arXiv:1705.03654 [gr-qc]. 